\title{
ERRATUM
}

\section{Regulation of prothymosin $\alpha$ by estrogen receptor $\alpha$ : molecular mechanisms and relevance in estrogen-mediated breast cell growth}

Nicole R Bianco and Monica M Montano

Oncogene (2002) 21, 8221. doi:10.1038/sj.onc.1205995

Correction to: Oncogene (2002) 21, 5233-5244. doi:10.1038/sj.onc.

The doi for the above paper was printed incorrectly. The correct doi is:

doi: $10.1038 /$ sj.onc. 1205645 